\title{
Participación ciudadana y acción gubernamental: una curva de indiferencia para la seguridad pública en México
}

\section{Citizen participation and government action: an indifference curve for public security in Mexico}

DOI: https://doi.org/| 0.32870/eees.v26i76.679|

Joaquín Ordóñez*

\section{Resumen}

Este trabajo explora teóricamente la relación existente en México entre seguridad pública, como variable dependiente, y los instrumentos por los cuales se obtiene:participación ciudadana y acción gubernamental, como variables independientes. Se consideran también los conceptos democráticos de coto vedado y ámbito de negociación. Se analizan dos escenarios hipotéticos: uno en que el Estado no cumple con el objetivo de proporcionar seguridad pública, y otro en que sí lo cumple. Se muestra que la relación de las variables es distinta en cada caso, ya que existe una curva de indiferencia en la que a veces el ciudadano obtiene la misma satisfacción y pierde interés por uno u otro instrumento, pues la utilidad que esto podría representarle es la misma en cualquiera de los casos.

Palabras clave: participación ciudadana, acción gubernamental, seguridad pública, democracia participativa, curva de indiferencia.

\begin{abstract}
This work explores theoretically the relationship in Mexico between public securityas a dependent variable, and the instruments by which it is obtained: citizen participation and governmental action -as independent variables. Democratic concepts coto vedado (forbidden limits) and ámbito de negociación (negotiating area) are also considered. Two hypothetical scenarios are analyzed: one in which the state does not meet the objective of providing public safety, and a second one in which it does. Conclusion shows that the relationship between variables is different, since there is a curve of indifference in which the citizen sometimes gets the same satisfaction and loses interest in one instrument or another, because the utility that this could represent to him is the same in any case.
\end{abstract}

Keywords: Citizen participation, governmental action, public security, participatory democracy, curve of indifference.

\footnotetext{
- Profesor-Investigador de la Facultad de Derecho de la Universidad Autónoma del Estado de México (UAEMEX), México. ORCID: https://orcid.org/0000-0002-6447-7I88

joaquin.o@me.com

Fecha de recepción: 05 de febrero de 2017. Fecha de aceptación: 17 de junio de 2019.
} 


\section{Introducción}

La seguridad pública es un bien social que se sostiene con la acción de diversos agentes sociales, entre los cuales están el Gobierno y la ciudadanía, ambos como elementos estatales que se encuentran en una continua tensión que depende de la participación ciudadana y de la acción gubernamental. Por ello, la participación y la acción se erigen en un verdadero instrumento para el logro de aquel bien.

En México, la seguridad pública es un problema que desde hace algunos años ha rebasado las capacidades gubernamentales e institucionales y provocado diversas formas de intervención ciudadana para contrarrestarlo; por ello, la finalidad del presente trabajo es explorar teóricamente la relación existente entre diversos niveles de satisfacción de la seguridad pública como variable dependiente, por un lado, y la participación ciudadana y la acción gubernamental como variables independientes, por el otro, para lo cual se reflexionó acerca de la tensión entre la participación ciudadana y la actividad del Gobierno que se han desplegado para lograr la seguridad pública.

En un ambiente democrático en el que prevalece la inseguridad pública a causa de la deficiente acción gubernamental, el llamado coto vedado ${ }^{1}$ se flexibiliza, ya sea para extender los límites o para restringirlos respecto a la actividad de la ciudadanía o del Gobierno, y con el objetivo de combatir esa inseguridad. Esto hace que se vea afectado el ámbito de negociación política, tanto ciudadana como gubernamental, también ampliándose o reduciéndose.

En tal escenario, la participación ciudadana es un elemento necesario para alcanzar el objetivo de la seguridad

I. Como se verá más adelante, el coto vedado se refiere a una de las aristas de la democracia que prevé un ámbito constitucional en el que se incluyen principios y valores y se resguardan derechos fundamentales no negociables. Este concepto es ampliado posteriormente en el desarrollo de este artículo.

\section{8}


pública, por lo que a mayor flexibilidad del coto vedado, mayor amplitud del ámbito de negociación y mayor seguridad, y viceversa, a menor flexibilidad del coto vedado, menor amplitud del ámbito de negociación y menor seguridad. Por esa razón, es inevitable considerar al coto vedado y al ámbito de negociación como otras variables independientes.

Contrario a lo anterior, en un escenario en que el Estado cumple con proporcionar seguridad pública, la relación entre las variables cambia: a mayor seguridad pública, menor amplitud del ámbito de negociación.

Los dos escenarios planteados, aunados a las posibles preferencias del ciudadano razonable respecto a los instrumentos para alcanzar la seguridad pública, provocan una curva de indiferencia, ya que en algunos casos el ciudadano obtiene el mismo grado de satisfacción respecto a la seguridad pública y pierde interés por involucrarse en las actividades estatales que buscan lograrla porque la utilidad que esto podría representarle es la misma con la utilización de cualquiera de los instrumentos referidos.

El presente artículo busca describir la relación entre los elementos planteados y la formación de dicha curva de indiferencia. En cuanto a la metodología usada para el estudio, se utilizó información estadística y periodística del periodo 2012 a 2017 en México, en concreto respecto del surgimiento de los grupos de autodefensa y de policías comunitarias, de la participación de la ciudadanía en ellos y de la deficiente intervención del Estado mexicano por conducto de sus órganos e instituciones gubernamentales para combatir la delincuencia y la inseguridad pública, lo anterior para establecer la relación entre la participación ciudadana y la acción gubernamental respecto de la seguridad pública. 


\section{Seguridad pública: un bien social como producto ciudadano y estatal}

El vocablo seguridad remite a la cualidad de seguro, que a su vez hace referencia a aquello que está libre o exento de riesgo, que es cierto e indubitable, que se encuentra firme o sujeto, que no falla o que ofrece confianza y certeza (Real Academia Española, 2017a). Público, por su parte, hace referencia a aquello que es conocido o sabido por todos o que se hace a la vista de todos, que es accesible y destinado para todos, y también a lo que es perteneciente o relativo al Estado (Real Academia Española, 2017b). La seguridad pública es, pues, un servicio que debe ser otorgado por el Estado $^{2}$ con la finalidad de que la población goce de tranquilidad y pueda tener una vida exenta de todos aquellos riesgos que lesionan o que potencialmente podrían lesionar su integridad física, moral, económica, jurídica, etc.

El impacto de la seguridad pública se encuentra en que gracias a ella los ciudadanos de un Estado tengan la posibilidad de coexistir en congruencia con los principios éticos y morales y en concordancia con el respeto al derecho de las demás personas. Por ello, se le define también como una cualidad de los espacios públicos y privados caracterizada por la inexistencia de amenazas que socaven o supriman los bienes y derechos de las personas y en la que existen condiciones propicias para la convivencia pacífica y para el desarrollo individual y colectivo de la sociedad (García Ramírez, 2002, p. 81).

Desde el punto de vista fenomenológico, se ha aceptado la descripción del Estado bajo los siguientes elementos: "1. Una sociedad humana, 2. Establecida permanentemente

2. El noveno párrafo del artículo 2 I de la Constitución dice: "La seguridad pública es una función del Estado a cargo de la Federación, las entidades federativas y los Municipios, cuyos fines son salvaguardar la vida, las libertades, la integridad y el patrimonio de las personas, así como contribuir a la generación y preservación del orden público y la paz social [...]" (Diario Oficial de la Federación, 2019). 
en un territorio, 3. Regida por un poder supremo, 4. Bajo un orden jurídico, 5. Y que tiende a la realización de los valores individuales y sociales de la persona humana [...]" (González Uribe, 1995, p. 162); el último elemento mencionado, la realización de los valores individuales y sociales de la persona humana, significa que el Estado tiene, entre otras, la función de proporcionar y garantizar la continuidad de la seguridad pública, ya que sin ella no es posible que exista tal realización de valores de la persona.

$\mathrm{Al}$ respecto, es ilustrativo el Pacto de San José o Convención Americana de Derechos Humanos (CADH) (1969), que en su parte primera (alusiva a los deberes del Estado y derechos protegidos), artículo 7 , párrafo 1, establece que "Toda persona tiene derecho a la libertad y a la seguridad personales". ${ }^{3}$ Para el pacto, el Estado, en su carácter de responsable de la procuración de la seguridad pública, tiene a su disposición toda una estructura gubernamental sistematizada y ordenada que cuenta con diversas instituciones cuyo principal objetivo es lograr el cumplimiento de las metas estatales constitucional y legalmente establecidas.

Sin embargo, en México, la realización de valores individuales y sociales de la persona, traducida en seguridad pública, ha sufrido una crisis, ya que el Estado mexicano no ha cumplido con su objetivo, si se considera que las tasas de incidencia delictiva han ido en aumento (Instituto Nacional de Estadística y Geografía, 2017). ${ }^{4}$ Aunado a lo anterior, se debe considerar que en México, como resultado de esos

3. Incluso, la $\mathrm{CADH}$ hace depender otras libertades de las limitaciones prescritas por la ley que sean necesarias para proteger la seguridad pública, como la libertad de conciencia y de religión (artículo 12), la libertad de pensamiento y de expresión (artículo I3), el derecho de reunión (artículo I5), la libertad de asociación (artículo 16) y el derecho de circulación y de residencia (artículo 22).

4. Tasas de incidencia delictiva de ocurrencia, es decir, el número de eventos individuales de victimización delictiva reportados por cada cien mil habitantes:a) año 20 I O/I, 30535 eventos; b) año 20 I I/2, 29200 eventos; c) año 20 I 2/3, 35 I 39 eventos; d) año 20I3/4, 4I 563 eventos; e) año 20।4, 4I 655 eventos; y f) año 20I5, 35497 eventos.

Estado No.76 
fenómenos de inseguridad pública, hay un aumento en la desconfianza entre los propios ciudadanos ${ }^{5}$ y entre estos y las autoridades e instituciones del Estado cuya función sería concretar la seguridad pública.

Esto último se demuestra con los datos de la calidad de la ciudadanía en aspectos como la cultura de la legalidad, que incluye la opinión favorable que tienen los ciudadanos hacia la captura de presuntos delincuentes, el respeto de los derechos humanos por parte de las autoridades, u otros aspectos como el respeto a las leyes y las denuncias de los delitos, así como la eficacia de las autoridades que reciben tales denuncias (Instituto Nacional Electoral, 2015).

La idea de que la ciudadanía sea corresponsable de la seguridad pública puede ser contradictoria, en principio, respecto al elemento de realización de los valores individuales y sociales de la persona humana que, como ya se dijo, se incluye en lo que la teoría ha aceptado como propio del Estado, más aún si consideramos a la seguridad como un bien común que debe ser tutelado y garantizado estatalmente, idea ya desarrollada por Hobbes (1984, p. 302) al plantear en su teoría contractualista que el hombre debe asegurar su permanencia y conservación en el mundo por medio del cumplimiento de la ley natural, misma que ordena a los hombres buscar la paz a través de la defensa de quienes se les oponen con base en un contrato que garantice la armonía y en el que cada persona prescinda de algunos derechos, con todos en sociedad dispuestos a hacer lo mismo.

5.Así, dependiendo del nivel de satisfacción que proporcione la seguridad pública, para efectos del presente artículo esta será graduada en términos de su calidad/ cantidad, en una escala de nueves posiciones: $9=$ perfecta/total; $8=$ excelente/ mucha; $7=$ muy buena/bastante; $6=$ sobresaliente/suficiente; $5=$ buena/regular; 4=defectuosa/poca; 3=mala/insuficiente; $2=$ pésima/muy poca; $y$ I=nefasta/nada. Esas nueve posiciones corresponden a las nueve combinaciones de preferencias ciudadanas respecto a la participación ciudadana y la acción gubernamental para la satisfacción de la seguridad pública.

\section{2}


Hobbes señala que para que ese pacto se respete se requiere justicia y, además, una autoridad con el suficiente poder para asegurar esa cooperación pacífica basada en la renuncia a los derechos que se tenían en el estado de naturaleza, esto a través de un contrato que implique otorgar tales derechos a un soberano que garantice el orden y la seguridad. Cumplir con la ley que ordena a los hombres buscar la paz implica satisfacer el objetivo de asegurar la conservación y permanencia del hombre en el mundo, es decir, se constituye en un satisfactor.

Ahora bien, el Estado ha sido incapaz de cumplir con dicha encomienda social, lo que lleva a considerar la posibilidad de que esa responsabilidad estatal sea, si no relevada, al menos coadyuvada por otros elementos de la sociedad, como la ciudadanía. A raíz de ello, los instrumentos propicios para satisfacer el bien denominado seguridad pública son la participación ciudadana y la acción gubernamental.

Por otro lado, los eventos y fenómenos relacionados con la inseguridad pública proporcionan información acerca de lo multivariado que puede llegar a ser el cumplimiento de la seguridad pública como objetivo, y con ello de lo complejos que pueden ser los procesos realizados por el Estado, tanto que, al final, llevan a una conclusión: la seguridad pública debe ser un objetivo cuyo cumplimiento dependa no solamente de las acciones gubernamentales (con mayor razón en un Estado cuyo cumplimiento del objetivo se ha dificultado), sino de otros elementos estructurales y multifactoriales que incluyen, por ejemplo, la eficacia de la policía (tanto federal como estatal y municipal), la organización y funcionamiento de Poder Judicial, y también la participación ciudadana. En esas circunstancias, la relación que se crea entre la seguridad pública como bien social y los elementos productores de la misma demuestra la relación existente entre la seguridad pública como variable dependiente y la

Estado No.76 
participación ciudadana y la acción gubernamental como variables independientes.

También, las consecuencias de la participación ciudadana en un Estado democrático están imbricadas con otros conceptos democráticos, como el de coto vedado, el cual "puede ser definido, en términos generales, como aquel ámbito constitucional que incluye principios y valores cuyo respeto y/o implementación permite asegurar un funcionamiento cabal de la democracia representativa" (Garzón, 2003, p. 57), y que constituye los límites legales y constitucionales para la actuación de los órganos e instituciones gubernamentales y para el propio ciudadano.

De dicho concepto democrático se puede derivar el de ámbito de negociación, ya que es en "el concepto de 'coto vedado' en el que han de resguardarse los derechos fundamentales no negociables, como condición necesaria de la democracia representativa. Sólo fuera de este 'coto vedado' cabe el disenso, la negociación y la tolerancia" (Garzón, 2005, p. 57). Es decir, los conceptos señalan la existencia de dos zonas: una demarcada por los límites establecidos del coto vedado y otra que existe más allá de tales límites, y en la que hay la permisión de negociar por virtud de la actividad democrática -lo que no es posible en la zona configurada dentro de esa demarcación-.

Consecuentemente, si los límites establecidos por el coto vedado sufren alguna modificación (se flexibilizan, ya sea para extender o para restringir), entonces también la zona del ámbito de negociación sufre una reconfiguración (que puede ser de ampliación o de reducción). Lo anterior da pauta para abordar la participación ciudadana y la acción gubernamental como instrumentos sociales para la obtención de la seguridad pública como un bien, y para determinar la tensión existente entre ellos. 
Participación ciudadana y acción gubernamental: una curva de indiferencia para la seguridad pública en México

\section{Participación ciudadana y acción gubernamental: instrumentos sociales para la seguridad pública en una relación posible}

La sociedad requiere del cumplimiento de ciertas metas y objetivos que están dirigidos a saciar necesidades propias de sus integrantes (que, por ende, lo son también del Estado), las cuales van de las más básicas hasta las más complejas, como las relacionadas con algunos de los derechos de nuevas generaciones. Esto va en función del desarrollo (económico, político, social, etc.) del propio Estado, y marca la pauta en términos de las necesidades de la población (donde también inciden aspectos geográficos, climatológicos, territoriales, demográficos, ideológicos, etc.).

Sin embargo, saciar esas necesidades es solamente el principio en cuanto a los propósitos estatales, ya que también se requiere dar solución a las dificultades y problemas que se van presentando (de tipo social y de competencia estatal), y satisfacer ciertas exigencias que eventualmente surgen. Por ello, la acción gubernamental se erige en un instrumento para la satisfacción de ciertos bienes sociales, como la seguridad pública. No obstante, también la participación ciudadana puede ser un elemento social con la capacidad de satisfacer tales necesidades.

La utilidad y el beneficio son las pautas que se enmarcan dentro de la comprensión conceptual de un bien, ya que no se podría concebir su contenido conceptual en un sentido diferente: los bienes ${ }^{6}$ no podrían (no deberían) constituir un perjuicio sino un efecto a su acreedor, esto respecto de su valor positivo intrínseco, que por lo mismo es estimable y no, por el contrario, desestimable o indeseable; en una alusión

6. Aquí, el concepto de bien excluye a aquellos objetos o sujetos de la realidad que en su propia esencia poseen la cualidad de la perfección (o se acercan a ella) como un complemento de sus características que los convierte en los mejores respecto al género de cosas al que pertenecen.

Estado No.76 
a la conceptualización de la economía, se hace referencia como bien a todo aquello que es apto para satisfacer una necesidad humana, ya sea de manera directa o indirecta (Real Academia Española, 2018). Por tales motivos, la seguridad pública se erige, per se, en un bien, aunque también podría ser un satisfactor, pues puede proporcionar la satisfacción de otras necesidades humanas, como las que atañen a los diversos derechos fundamentales que, al final, otorgan tranquilidad y paz a los ciudadanos -y habitantes en general- de un Estado.

Así, la seguridad pública es un bien social que se constituye en una variable dependiente, pues es producida tanto por la participación ciudadana como por la acción gubernamental, mismas que, a su vez, se erigen en variables independientes. También, como ya quedó establecido, de la participación ciudadana se derivan los diversos grados de flexibilidad del coto vedado como límite jurídico establecido para la actividad tanto del Estado (traducida en acción gubernamental) como de la ciudadanía (traducida en participación ciudadana), y que puede adoptar tres graduaciones: flexible para extender, inflexible o rígido, y flexible para restringir.

Aunado a lo anterior, el concepto de ámbito de negociación hace referencia a la exclusión de algunos temas básicos de la negociación parlamentaria o de la votación ciudadana, ya que "deben ser excluidos de la negociación y el compromiso parlamentarios todos aquellos bienes que son considerados como básicos para la realización de todo plan de vida" (Garzón, 2005, pp. 21-22); por tanto, el ámbito de negociación se comporta en función de la flexibilización que del coto vedado haga la participación ciudadana, y puede tornarse amplio, igual o reducido, pues existe una relación de dependencia entre uno y otro: si los límites se flexibilizan para extender, entonces el área de negociación se ensancha; 
si se mantienen inflexibles o rígidos, entonces se mantiene igual; y si se flexibilizan para restringir, entonces se reduce.

Por otro lado, en el contexto estatal, la participación ciudadana se erige como un instrumento satisfactor de la seguridad pública, como un bien en virtud de la importancia con la que intervienen participación y Estado en el funcionamiento de la sociedad: "La existencia de instituciones sin participación supone el riesgo de meros aparatos legales formales carentes de vida. La participación sin instituciones supone el riesgo de una simple protesta en el vacío" (Beliz, 2012 , p. 22). La participación es (o debe ser) una cualidad del ciudadano, es decir, participación y ciudadanía deben ser consideradas como un binomio inseparable, al grado de que no debería ser posible concebir a una sin la otra.

Ahora bien, antes de continuar con la reflexión acerca de la participación ciudadana, se hace indispensable considerar al ciudadano desde la arista relacionada con sus cualidades racionales, es decir, el ciudadano razonable. $\mathrm{Al}$ respecto se debe considerar que:

Son los individuos, hombres y mujeres, quienes garantizan el mantenimiento de un determinado orden político a través de sus gustos, creencias, conocimientos y costumbres; pero sólo cuando todo esto proviene de su libre deliberación y decisión. Por eso cada régimen político fomenta en sus ciudadanos los valores sobre los cuales se sostienen. Así [...] la república [...] necesita ciudadanos razonables y respetuosos de las leyes fundadas en el interés de salvaguardar y promover los derechos de todos. Eso requiere que cada ciudadano tenga conocimiento claro de lo que debe competir a la ley, y una opinión razonable acerca de las circunstancias históricas en las que vive. (Carrillo, 20I0, p. II5)

El funcionamiento de un Estado recae sobre los ciudadanos y, por tanto, dependiendo de las características de los ciudadanos será su funcionamiento, por lo que una república democrática necesita que sus ciudadanos conozcan y 
practiquen la democracia, y esto se logra siendo racionales y por medio de la educación: "el sistema educacional tiene [...] un claro objetivo moral, cuya realización es la producción de un determinado tipo de ser humano: un ciudadano razonable que ejerce sus derechos" (Carrillo, 2010, p. 115).

El ciudadano razonable es, por tanto, aquel cuya conducta es adecuada a la razón, considerando a esta como un criterio de moralidad que debe sustentar la forma de pensar de quien la posee, lo que a su vez obliga al ciudadano a comportarse de una determinada forma e incluso lograr ciertos acuerdos: "La idea del ciudadano razonable, capaz de renunciar a la imposición unilateral de su concepción de lo bueno, parecería ser una buena solución para asegurar la obtención de acuerdos democráticos en sociedades populosas y culturalmente heterogéneas" (Garzón, 2011, p. 77).

En esa concepción de razonabilidad, se ve involucrado inevitablemente el concepto de moral, ya que implica una disposición ciudadana de actuar moralmente: "La disposición a ser razonable no se deriva de ni se opone a lo racional[,] pero es incompatible con el egoísmo, porque está relacionada con la disposición a actuar moralmente" (Rawls, 1993, p. 49).

Esa concepción de razonabilidad como cualidad del ciudadano también puede ser considerada en un conjunto, como una sociedad civil, cuya actividad estatal implica la transferencia de la calidad moral al propio sistema político, pues la razonabilidad significa también un contrapeso o una contrapartida que obstaculiza al egoísmo como especie conductual del ciudadano y del funcionario público. A esto, la tradición lo ha llamado imposición incondicionada de las propias preferencias (Garzón, 2011, p. 78), y también ha sustentado que un Estado democrático cuya población esté conformada por ciudadanos razonables podría alcanzar el más alto nivel de justicia y tendría su legitimidad surgida directamente de la disposición para actuar moralmente. Por 
todo esto es que el concepto de ciudadano razonable debe ser considerado para efectos del presente artículo.

Ahora bien, el resultado del ejercicio de la participación ciudadana se puede medir en función de los bienes que proporciona al ciudadano, es decir, el ciudadano tiene la posibilidad (y también mayor probabilidad) de que las metas y finalidades del Estado sean cumplidas a cabalidad con la consecuente obtención de los beneficios gracias a la acción ciudadana de tomar parte en los asuntos públicos de un Estado.

No obstante, en México la participación ciudadana alcanza poca intensidad, ${ }^{7}$ lo que va aparejado a la poca obtención de algunos de los ya comentados bienes y, por tanto, es un indicativo del poco aprovechamiento de la participación en su carácter de instrumento de satisfacción social. Esto se suma a lo evidenciado por la opinión pública mexicana: en quien más desconfía la ciudadanía es en los políticos que gobiernan el país, seguidos de la policía (Centro de Opinión Pública e Instituto para la Seguridad y la Democracia, 2017), además de que la corrupción sigue siendo una de las principales razones por las que la gente desconfía de la policía (54\% de opiniones al respecto), se percibe abuso policial (28\%), se han tenido malas experiencias personales o de alguien cercano con la autoridad de seguridad (19\%), se considera a la policía vinculada con grupos criminales (13\%), o se le relaciona con falta de capacitación o no ejecución adecuada de su labor (11\%), todo lo cual la vuelve desconfiable.

Así las cosas, y considerando que la seguridad pública es un bien producido por la acción gubernamental pero también por la participación del ciudadano, como ya se dijo, y que estos dos últimos elementos se constituyen en instru-

7. Por ejemplo, en las elecciones presidenciales de los años 2000 y 2006 hubo una baja en cuanto a participación, mientras que en las de 2012 se observó un aumento con una tasa del $62.08 \%$, lo que representa un crecimiento de 3.85 puntos con respecto al 2006 (Instituto Federal Electoral, 20I3).

Estado No.76 
mentos sociales que producen determinados bienes de tipo fundamental, ${ }^{8}$ se puede delimitar ahora cuantificablemente cada uno de esos elementos para analizar la relación entre la acción gubernamental y la participación ciudadana de modo que sea posible vincularlos en varias combinaciones relativas a las preferencias del ciudadano razonable.

Ya se ha dicho que tanto la acción gubernamental como la participación ciudadana son fundamentales para el logro de la seguridad pública al interior de un Estado, ${ }^{9}$ pero es importante recalcar que el ciudadano es corresponsable de ese bien al tener la carga de los deberes que debe cumplir en relación con su familia, con la sociedad y con la comunidad global, en términos de la estructura democrática. $\mathrm{Al}$ respecto, el artículo 32 de la $\mathrm{CADH}$ señala que "1. Toda persona tiene deberes para con la familia, la comunidad y la humanidad. 2. Los derechos de cada persona están limitados por los derechos de los demás, por la seguridad de todos, y por las justas exigencias del bien común, en una sociedad democrática" (Convención Americana de Derechos Humanos, 1969).

Dicho esto, en México hay casos de organización ciudadana para el combate a la delincuencia que consisten en la realización de simples advertencias por medio de mensajes escritos y visibles en las calles, dirigidos a los delincuentes, lo cual es una manera de ampliar la actividad y la responsabilidad de los ciudadanos por voluntad propia, ampliación

8. Aquellos sin los cuales no podría afirmarse que el individuo vive una vida plena, desde luego, dentro de lo razonablemente limitado por otros factores físicos e ideológicos.

9.Y surge la siguiente interrogante: ¡cuál es la cantidad de acción gubernamental requerida para solucionar el problema social de la inseguridad pública? $\mathrm{O}$, dicho de otra manera, iqué cantidad de acción gubernamental podría asegurar la solución a una cierta cantidad de inseguridad pública? También sería necesario cuestionar acerca del parámetro por el cual se debería/podría realizar la medición de acción gubernamental y de participación ciudadana. Con lo anterior, se podría determinar la existencia o no de un equilibrio en función de las cantidades tanto de acción gubernamental como de participación ciudadana. Tales cuestionamientos podrían ser materia de otra investigación, ya que están fuera del propósito de este artículo.

\section{0}


que si bien está sustentada en el deber de cada uno de los ciudadanos respecto a su familia y su comunidad, no tiene un fundamento jurídico para su ampliación -lo que se ha visto en algunos casos-, aunque, en contraposición, el fundamento moral sea un fundamento plausible en ella.

Es por esto que dicha ampliación es en realidad una manera de participar de la ciudadanía cuyo efecto va en detrimento de la propia actuación gubernamental y de las atribuciones formal y jurídicamente establecidas para el Gobierno en diversos cuerpos normativos. ${ }^{10}$ El resultado de lo anterior es una sociedad democrática en la que la actividad de una persona o de un ciudadano repercute en la esfera de libertades e igualdades de las otras personas o ciudadanos, y donde una conciencia de masas se expresa para tomar determinaciones respecto a lo público.

Sin embargo, no solamente los mensajes contra delincuentes son casos en los que se puede evidenciar la participación del ciudadano en cuanto a la seguridad pública, sino que existen otros que están relacionados también con aspectos que implican una mayor organización por parte de la comunidad, ${ }^{11}$ y que llevan a la conclusión de que dicha participación ha llegado a límites aún más amplios y lejanos que, mientras acrecientan dicha participación, reducen

10. Estas formas de participación ciudadana podrían ser consideradas una manera de reaccionar por parte de la ciudadanía ante un andamiaje estatal apático e ineficaz respecto a sus deberes, y en un real ejercicio de la soberanía sustituyen, al menos, una parte de las funciones estatales respecto a la seguridad pública. Sin embargo, esta hipótesis excede los propósitos de este trabajo, pudiendo quedar como objetivo demostrarla posteriormente.

II. Por ejemplo: los sistemas de vigilancia privada, las redes de autoprotección como la vigilancia vecinal, los organismos no gubernamentales como México Unido contra la Delincuencia o los grupos de autodefensa michoacanos (Univisión, 2013), así como casos en los que se ha hecho justicia por propia mano, como el del Grupo de Élite Antirratas, banda que en octubre de 2016 amputó las manos a siete presuntos ladrones en Tlaquepaque, Jalisco (El Mundo Internacional, 20 I6), o el caso del llamado vengador anónimo, en que cuatro hombres fueron asesinados por un viajero del autobús que recién habían asaltado en noviembre de 2016 (Excélsior, 2016).

Estado No.76 
el ámbito de acción del sector gubernamental o, al menos, evidencian que es menor de lo que se cree.

La ciudadanía y el Estado están amalgamados e involucrados una con el otro, lo anterior en virtud de que en un Estado democrático y republicano lo importante es la cosa pública (el objeto de interés de todos), lo cual se traduce y se ha traducido -en mayor o menor medida- en tomar parte una del otro, es decir, el Estado se constituye como parte integral de la ciudadanía y la ciudadanía del Estado, ya que este último está conformado por seres humanos que hacen funcionar sus órganos e instituciones y esos seres humanos son a su vez ciudadanos (considerando que para poder ocupar esos cargos o puestos públicos deben cumplir con ciertos requisitos mínimos, entre los cuales está la ciudadanía y otros derivados de ella), lo cual lleva a la conclusión de que la ciudadanía y el Estado son una entidad que comparte ciudadanos.

No obstante, existe un punto -que puede válidamente tomarse como referencia- en el que el Estado y la ciudadanía se distinguen uno de la otra. Se trata de un punto en el que no es posible que sus cualidades se mezclen en una especie de coparticipación de la cosa pública (en virtud de una distinción precisa derivada de su formalidad constitucional y legal), y esto se evidencia en las funciones, atribuciones, estructuras, etc., mismas que no deben mezclarse ni confundirse, y que están establecidas tanto en la normatividad legal como en la constitucional y no permiten que dichas atribuciones sean ejercidas -al menos desde el punto de vista de la formalidad-por uno u otro de estos entes de manera indiscriminada. Lo anterior lleva a la conclusión de que existe una división clara respecto a la ciudadanía al menos en esos aspectos formales que lleva a considerar una plausible área de ejercicio de uno y otro.

Esto es, la acción gubernamental se traduce en una intervención de los órganos e instituciones estatales, mien-

\section{2}


tras que la participación ciudadana tiene un efecto en el involucramiento de la población en cuanto a la seguridad pública. Los anteriores elementos están en continua tensión en función de la flexibilidad del coto vedado y de la amplitud del ámbito de negociación, pues a pesar de que los órganos e instituciones del Estado están compuestos e integrados por ciudadanos existe un límite (coto) a la actuación tanto de esos órganos estatales como de la ciudadanía, ${ }^{12}$ el cual marca la división entre las funciones y atribuciones constitucionales y legales de los órganos e instituciones del Estado y de los derechos y obligaciones democráticos de los ciudadanos, también derivados de la constitucionalidad y legalidad. Sin embargo, en un ambiente en el que la seguridad pública no es la adecuada, esos límites (y, consecuentemente, el ámbito de negociación) se ven flexibilizados, con el efecto de que en ocasiones el ciudadano podría estar realizando actividades que jurídicamente no le corresponden por ser atribución de los órganos e instituciones del Estado.

En efecto, en una sociedad democrática, la amalgama entre Estado y ciudadano se evidencia con mayor claridad en la toma de decisiones por virtud del instrumento de la mayoría, el cual otorga legitimidad al propio sistema (además de dar solución a problemas sociales):

los procedimientos políticos deberían ser diseñados para que [...] la decisión alcanzada sea la que favorece a una mayoría o pluralidad de ciudadanos, [...] las leyes que dicta el complejo proceso democrático y las políticas que persigue deberían ser aquellas que, finalmente, la mayoría de los ciudadanos aprobaría. (Dworkin, 2004, pp. I I5 y I I6)

12. El cual está sustentado por el principio de legalidad: los funcionarios públicos (ciudadanos integrantes de los órganos o instituciones del Estado) solamente pueden hacer lo que la ley les ordena, mientras que los ciudadanos (fuera de su posible función dentro de los órganos e instituciones estatales) tienen la posibilidad de hacer todo lo que la ley no les prohíbe.

Estado No.76 
Cuando la decisión alcanzada favorezca a la mayoría de los ciudadanos y se haya logrado gracias a procedimientos estatales que tiendan a ello, se habrá logrado también el necesario involucramiento entre la acción gubernamental y la participación ciudadana con miras a un potencial equilibrio entre una y otra. Desde luego que tal equilibrio debe tener como efecto la utilidad, ya que no sería razonable que en un Estado democrático donde se amalgama la acción gubernamental con la participación ciudadana las decisiones tomadas por la mayoría no beneficien a esa misma mayoría:

La justificación utilitarista obvia de la regla de la mayoría descansa sobre la posibilidad de alcanzar un determinado valor social (felicidad general, bienestar, utilidad, etc.) incluso cuando este se logre como consecuencia del autointerés de los individuos. (Santiago, 2003, p. 107)

Ahora bien, la posibilidad de una relación entre acción gubernamental y participación ciudadana se da por la intensidad de las preferencias de la propia ciudadanía en contraste con los mecanismos para hacerlas valer -o expresar- del Estado (como la regla de la mayoría):

la regla de la mayoría por sí misma no satisface el principio utilitarista, ya que para maximizar las preferencias deben ser evaluadas sus intensidades[;] la regla de la mayoría simple puede tener resultados antiutilitaristas si los intereses de la mayoría son mucho menos intensos que aquellos de la minoría. (Santiago, 2003, p. 107)

El aspecto utilitario de que la acción gubernamental y la participación ciudadana se desarrollen bajo una perspectiva de relación está sustentado en la necesidad de que la población sea tomada en cuenta respecto a los programas sociales y a las políticas democráticas destinadas al bienestar general de la población:

\section{4}


la participación de masas es un elemento indispensable para toda comunidad democrática. En su ausencia, los líderes se tornan irresponsables ante las necesidades de las personas. Al extenderse la brecha entre gobernantes y gobernados, las masas pueden tornarse alienadas, desentendidas y mezquinas $y$, al suceder esto, se expone a la democracia a las arremetidas de movimientos de masas antidemocráticos. Lo que es más importante, una "democracia" que elimine la participación de masas llegando al grado de promover su no participación niega a los estratos más grandes de la población los beneficios derivados de la participación política-socavando así su principal razón de ser, vale decir, la promoción del bienestar general-. (Bachrach y Botwinick, 1994, pp. I 36 y I37)

Por ello, no solamente la acción gubernamental está justificada en la toma de decisiones al interior de un Estado, sino que también es necesaria la participación de la ciudadanía como una parte activa de la gestión pública:

la participación ciudadana es una forma de entender el ejercicio del poder donde los sujetos, entendiendo por tal a los ciudadanos, forman parte activa de la gestión pública [...][;] la participación ciudadana es un derecho ciudadano fundamental, configurándose como un prerrequisito para el ejercicio de otros derechos. (Mujica, 2008, p. 22)

Consecuentemente, también la participación ciudadana y la acción gubernamental, en su carácter de instrumentos sociales (esto es, como ya se dijo, por los cuales se obtienen otros bienes, como la seguridad pública), constituyen variables independientes. Respecto a la manera en que los ciudadanos pueden realizar la actividad de participación en el Estado, algunos estudios (Mujica, 2008, pp. 24-35) abordan lo que llaman mecanismos modernos de participación, los cuales van más allá de los clásicos instrumentos representativos y se han clasificado según las acciones que se realizan desde las instituciones, que pueden ser: 1) participación en la definición, ejecución y evaluación de la gestión pública; 2) 
participación en programas presupuestales; 3) mecanismos de debate; y 4) encuestas deliberativas.

También, dichos estudios mencionan el control ciudadano basado en: 1) disposición de recursos sancionadores para el ejercicio del control; 2) el derecho a la información y los espacios de discusión; y 3) habilitación de los sujetos de control ciudadano. Finalmente, respecto al grado de incidencia de la participación, esta puede ser: 1) consultiva, 2) decisoria (la ciudadanía da una opinión vinculante), y 3) como cogestión, esta última considerada como el grado de influencia máxima de la sociedad civil por su intervención en los procesos de diseño de política pública, en sus etapas tanto de ejecución como de evaluación (Mujica, 2008, pp. 33 y 34).

Al final, la participación ciudadana y la acción gubernamental se erigen en innegables instrumentos sociales cuya mejor utilidad es el logro de la seguridad pública, y evidencian un binomio en relación de interdependencia. El siguiente paso es analizar esa relación a la luz de las hipotéticas, pero plausibles, preferencias de un ciudadano razonable con respecto a dicho binomio en dos contextos sociales: uno en el que el Estado no cumple con su función de proporcionar seguridad pública, y otro en el que sí la cumple.

\section{Participación ciudadana y acción gubernamental en una curva de indiferencia}

El ciudadano como parte de la población, y esta a su vez como elemento de un Estado, es en quien recae la actuación del Gobierno. Como ya se dijo, una finalidad estatal es proporcionar satisfactores a la población, por tanto, es plausible esperar que el ciudadano posea determinado nivel de satisfacción respecto al disfrute de los bienes que son consecuencia de la acción gubernamental, por lo que, respecto al problema abordado en este artículo, dependiendo de tal acción y de la cantidad de seguridad pública (entendida

\section{6}


como un bien) que el Estado produzca, se puede esperar nula o total satisfacción.

De lo anterior se sigue que sea necesaria la cuantificación en materia de seguridad pública y de satisfacción para determinar si el Estado es cumplidor de ese objetivo, así como la conformidad o no del ciudadano respecto a lo dicho. En caso que el Estado no cumpla, la cuestión es: ¿qué tan amplio tiene que ser el ámbito de negociación, a causa de la flexibilización del coto vedado por la actuación del ciudadano en sustitución del Estado, para que el ciudadano considere a este como satisfactor de los problemas de inseguridad? Y, por tanto: ¿en qué punto en la búsqueda del equilibrio/ relación entre la participación ciudadana y la acción gubernamental ocurre una curva de indiferencia que señale que se le proporciona a la ciudadanía la misma utilidad si la solución de dichos problemas está en la actuación de una y otra (participación ciudadana y acción gubernamental)?

Para resolver lo anterior, se deben considerar dos escenarios hipotéticos: en el primero, se plantea un Estado cuya acción gubernamental no es suficiente para el combate a la delincuencia, esto es, no es capaz de proporcionar seguridad pública a sus miembros; en el segundo, se plantea un Estado que sí cumple cabalmente con ese objetivo.

En el caso mexicano, el fenómeno de las autodefensas que desplazan al Gobierno y asumen la seguridad es un evento provocado por la mala administración de los órganos estatales:

Primero tomaron las armas, luego impusieron un "toque de queda" $y$ ahora la guardia ciudadana surgida desde hace un mes [...] decide confrontar directamente a la delincuencia ante la indolencia gubernamental. "Ya estábamos hartos de los asesinatos, secuestros y extorsiones que realizaba La Familia, el grupo que controlaba Apaxtla, pero ahora ya estamos más tranquilos", expresa un miembro de las autodefensas [...]. Al respecto, el alcalde perredista de Apaxtla de Castrejón, Efraín Peña 
Damasio, dijo que no se trata de un movimiento armado, más bien es, dice, la muestra del "hartazgo ciudadano" ante la omisión del Gobierno federal y estatal que desestimó los llamados de auxilio lanzados desde el año pasado, cuando el edil asumió el cargo en medio de una grave crisis de inseguridad y violencia. (Flores, 2013)

Las autoridades estatales también han hecho referencia al fenómeno. Por ejemplo, el entonces secretario de Gobernación mexicano, Miguel Ángel Osorio Chong, dijo que "Al final cada quien se encargará de lo que le corresponde: el Estado mexicano se hará responsable totalmente de la seguridad [...] el problema de inseguridad se ha reducido en setenta por ciento" (Martínez Elorriaga, 2014).

Las declaraciones de las autoridades hacen referencia no solamente al surgimiento de las autodefensas, sino también a la incapacidad del Estado mexicano para responsabilizarse de la seguridad, lo que demuestra que la acción o inacción del Gobierno y sus consecuencias son reconocidas incluso por sus mismos actores.

$\mathrm{Al}$ respecto, existen otras declaraciones que identifican la debilidad del Gobierno derivada del vacío que provocan las administraciones interinas, por ejemplo: "2015 fue un año particularmente difícil para Guerrero. Hubo un Gobierno estatal interino, extraordinariamente débil. El vacío de autoridad propició que se profundizaran los conflictos entre distintos grupos criminales y otros grupos armados" (Guerrero, 2016). Lo anterior corrobora el hecho de que los problemas de política o de administración pública perjudican la función primordial del Gobierno respecto a la seguridad pública, pero también que cuando este asume su responsabilidad y cumple con sus funciones se nota un cambio drástico respecto al fenómeno: "Michoacán [...] experimentó una disminución considerable de la violencia en 2014 debido a la intervención de las fuerzas federales" (Guerrero, 2016).

\section{8}


En ese último sentido, el fortalecimiento de las instituciones de seguridad ha resultado un factor determinante para demostrar que la intervención adecuada del Estado debería lograr el objetivo de proporcionar seguridad a la población:

De 2008 a 2010 las ciudades más afectadas por la violencia fueron Juárez, Tijuana, Culiacán, Monterrey, Torreón, Chihuahua, Durango y Reynosa. Varias de ellas han logrado fortalecer sus instituciones de seguridad, notablemente sus cuerpos policiales, y con ello y otras acciones de prevención social han logrado reducir la violencia. (Guerrero, 2016)

Morelos es un ejemplo destacado de una entidad con recursos limitados que padecía un problema severo de secuestro, pero que ha logrado reducirlo de forma dramática por medio de la operación de su unidad antisecuestro [...] [;] mientras en 2014 se registraron doscientos catorce plagios en Morelos, de enero a agosto de 2015 hubo treinta y cuatro. (Guerrero, 2016)

Sin embargo, esto corresponde a una estrategia gubernamental que solamente se ha implementado en algunos lugares; en el resto del país, el problema continúa: "Afuera de las ciudades las mafias extorsionan a empresarios de los sectores ganadero, agricultura y minería, principalmente, quienes en varios casos han optado por financiar 'grupos de autodefensa' para proteger a sus familias y su patrimonio" (Guerrero, 2016). La situación más constante, pues, es la escasa presencia del Estado respecto a la seguridad:

En enero de 2013 empezaron a aparecer grupos de civiles armados en distintas entidades del país [...] conocidos como "grupos de autodefensa", no solamente fueron una respuesta a las actividades delictivas de organizaciones criminales que afectaron los intereses de empresarios rurales (agricultores, ganaderos y mineros), sino la consecuencia natural de la escasa presencia que tiene el Estado mexicano en amplias franjas del país [...]. Para finales de 2014[,] los grupos de autodefensa 
en Michoacán tenían operaciones en treinta y tres municipios - $56 \%$ del territorio estatal-, mientras que en Guerrero estos grupos operaban en cincuenta $y$ tres municipios $-57 \%$ del territorio estatal-. (Guerrero, 2016)

Además de las ya señaladas autodefensas, otra vertiente que matiza el fenómeno de la participación ciudadana ante la inseguridad es la aparición de policías comunitarias, las cuales cuentan con algunas características especiales que las hacen ser un ejemplo también de cómo el Estado mexicano ha fallado con su labor institucional de controlar la delincuencia:

[...] poseen cuatro rasgos principales: I) los ciudadanos de una comunidad asumen labores de seguridad pública en sustitución de las autoridades estatales; 2) existe un apoyo o consenso comunitario que respalda el funcionamiento de estos grupos; 3 ) existe un reconocimiento formal o informal de las autoridades gubernamentales; 4 ) los grupos sustentan su actuación por medio de un entramado normativo. (Guerrero, 2016)

Independientemente de todas estas estrategias ciudadanas, como ya se vio, las condiciones mínimas de seguridad que deben surgir directamente de la labor institucional del Estado son el elemento primordial para el control del problema de la inseguridad, y por tanto, el desarrollo de las capacidades institucionales es indispensable. A ese respecto, el sistema federal de readaptación social es un elemento crucial, sin embargo, sucesos como las dos fugas del narcotraficante Joaquín "El Chapo" Guzmán Loera (2001 y 2015) han puesto en evidencia su defecto, ya que también se ha detectado que "se incrementó el número absoluto de fugas y la tasa de éxito de las mismas pasó del 6\% al 25\%" (Guerrero, 2016).

Lo anterior pone en evidencia que el Estado mexicano ha incumplido con su labor de garantizar la seguridad pública. 
Esto impacta, como ya se ha señalado, en la flexibilidad del coto vedado y en la ampliación o reducción del ámbito de negociación que deriva de ello. En ese escenario de deficiencia estatal es donde la participación ciudadana se convierte en un elemento indispensable para alcanzar el bien seguridad pública, ya que los límites formales impuestos a la sociedad y al propio Estado (por medio de instrumentos jurídicos tales como la constitución o las leyes secundarias) se constituyen a su vez en dicho coto vedado flexibilizado, y generan un medio social y estatal en el que esa maleabilidad de los límites jurídicos redunda en una ampliación del ámbito de negociación. Si los límites del coto (fuera del cual caben el disenso y la negociación) no cumplen con su objetivo de resguardar los derechos fundamentales, entonces tienen que flexibilizarse para lograr una mayor cantidad de seguridad pública con base en una mayor amplitud del ámbito de negociación.

No obstante, la ampliación del ámbito de negociación no es lo único que puede suceder en el fenómeno aquí estudiado, pues también es posible que en el desarrollo la participación ciudadana para el combate a la delincuencia dicho ámbito de negociación se vea reducido por el abuso en que potencialmente podría incurrir la ciudadanía al asumir parte del poder estatal en su ejercicio.

Por ello, el resultado de la participación ciudadana es una tensión entre el grado de cumplimiento del Estado en cuanto a su labor, por un lado, y el grado de intervención ciudadana, por el otro, y dicha tensión resulta en una competencia entre el Gobierno y la ciudadanía para lograr eficacia y utilidad social. Esto trae como resultado una variación en la calidad y cantidad de la seguridad pública en función del comportamiento estatal (acción de órganos e instituciones gubernamentales) y de la participación ciudadana.

Esto es por lo que, dependiendo del cumplimiento del Estado, surge la relación en la que a determinada canti- 
dad de acción gubernamental corresponde una determinada cantidad de participación ciudadana, de tal manera que, respecto del bien denominado seguridad pública, la acción gubernamental y la participación ciudadana son instrumentos para su obtención (junto a la flexibilización del coto vedado y la ampliación del ámbito de negociación consecuente, como ya se dijo) que pueden ser combinados de diversas maneras para también otorgar al ciudadano un determinado nivel de utilidad o satisfacción.

$\mathrm{Al}$ respecto, se pueden suponer diversas combinaciones de esos instrumentos en términos de preferencias ciudadanas, las cuales podrían ir de una mayor hasta una menor acción gubernamental o participación ciudadana, pasando por las diferentes combinaciones. Las combinaciones posibles de hipotéticas preferencias ciudadanas son las siguientes:

1. Mayor acción gubernamental y mayor participación ciudadana.

2. Mayor acción gubernamental e igual participación ciudadana.

3. Mayor acción gubernamental y menor participación ciudadana.

4. Igual acción gubernamental y mayor participación ciudadana.

5. Igual acción gubernamental e igual participación ciudadana.

6. Igual acción gubernamental y menor participación ciudadana.

7. Menor acción gubernamental y mayor participación ciudadana.

8. Menor acción gubernamental e igual participación ciudadana.

9. Menor acción gubernamental y menor participación ciudadana. 
Las combinaciones 1, 2, 4 y 7 son aquellas que el ciudadano razonable podría preferir en caso de que el Estado no cumpla con su objetivo de proporcionar la seguridad pública a la población. En la combinación 1, es por demás razonable que el ciudadano prefiera una mayor acción gubernamental, siempre que esto no implique incurrir en la falla de un Estado dictatorial que solamente abusaría del poder público en detrimento de la democracia y de la propia seguridad pública, o de una manera tal que su mayor intervención perjudicara en lugar de beneficiar.

Asimismo, una mayor participación ciudadana es una preferencia razonable, en virtud de la necesidad de que la población intervenga en la solución de la problemática de la seguridad pública, y esto hace flexible (extiende) el coto vedado para que los límites que impone no sean un obstáculo a la actuación del ciudadano, lo que a su vez amplía el ámbito de negociación.

En la combinación 2, el ciudadano prefiere que el Gobierno tenga una mayor intervención, con la salvedad mencionada, y en virtud de que la participación ciudadana preferida es igual el coto vedado se mantiene inflexible o rígido conservando los límites jurídicos en su posición original e igualdad en el ámbito de negociación. En este caso, el beneficio está dado solamente por la mayor intervención estatal en la problemática, y el hecho de que el ciudadano prefiera mantener su participación dentro de los límites originales se justifica debido a una mínima expectativa en que la acción gubernamental se realizará y concretará la solución a la problemática de inseguridad pública.

En cuanto a las combinaciones 4 y 7 , el ciudadano prefiere igual y menor acción gubernamental, respectivamente, junto a una mayor participación ciudadana, lo que se justifica debido a que pierde importancia que el Gobierno intervenga, esto como consecuencia de una legitimidad degradada. En 
estos dos casos, el coto vedado se vuelve flexible (se extiende) y el ámbito de negociación se torna amplio.

Respecto a la combinación número 5, el ciudadano razonable podría preferirla en caso de que el Estado cumpla con el objetivo de proporcionar seguridad pública a la población, ya que se plantea una igual acción gubernamental y una igual participación ciudadana, lo cual razonablemente puede ser preferible en caso de que no exista el problema de la inseguridad como consecuencia de la acción de los órganos de Gobierno, al grado de que una igual participación del ciudadano en esos asuntos no haría una diferencia sustancial para mejorar la situación que ya de por sí es buena. En este caso, el coto vedado es inflexible o rígido, ya que una igual participación ciudadana no implica una modificación a los límites establecidos para ampliar el ámbito de negociación, por lo que este último se mantiene igual.

Por otro lado, podrían existir con poca probabilidad otras combinaciones de preferencias, como la número 3 , la cual no es una preferencia del ciudadano razonable, ya sea en el caso del Estado cumplidor como del no cumplidor. En un ambiente de seguridad pública, una mayor acción gubernamental sería redundante, o incluso podría ser perjudicial por la posibilidad de que el Estado incurra en exceso o abuso en el ejercicio del poder -lo que sí podría lesionar los principios y prácticas democráticas y flexibilizaría el coto vedado para restringirlo-; una menor participación ciudadana, por su parte, sería innecesaria en ese caso por no haber una justificación adecuada para ello. Asimismo, tampoco en un Estado incumplidor la opción 3 sería razonable, pues para resolver la inseguridad se preferiría una mayor participación ciudadana en sustitución de la deficiente acción gubernamental. En ambas circunstancias, pues, el ámbito de negociación se vería comprometido para reducirse.

En la combinación número 6 , el coto vedado se vuelve flexible restringido en virtud de que una menor participación 
combinada con una igual acción gubernamental sí implica una modificación a esos límites que tiende a la restricción de los derechos del ciudadano y que trae como consecuencia un ámbito de negociación reducido. En este caso, se debe tener en cuenta que el ciudadano puede preferir no participar de manera voluntaria en la solución de la problemática de seguridad social, ello derivado del cabal cumplimiento por parte de los órganos de Estado encargados y por una potestad que tiene para decidir si interviene o no, lo cual está dentro del área de la toma de decisiones jurídicamente permitidas.

Por último, algunas combinaciones aunque son posibles no son necesariamente probables, pues dentro de la preferencia del ciudadano razonable no son recomendables, como 8 y 9 , que están en el extremo de una menor acción gubernamental y una igual o menor participación ciudadana, lo cual, en un Estado democrático, es perjudicial, salvo que hipotéticamente los problemas de seguridad social no existan y la intervención de los órganos estatales fuera tal que en lugar de beneficiar produjera un perjuicio. Por tanto, en cuanto al coto vedado, en los casos 8 y 9 se produce una inflexibilidad o rigidez que a lo sumo podría producir un mantenimiento del estado de cosas junto a un ámbito de negociación igual, ${ }^{13}$ pero que en el extremo de la combinación de preferencias produciría una flexibilidad restringida y una reducción al coto vedado y al ámbito de negociación, respectivamente.

Los dos grupos de combinaciones de preferencias ciudadanas en un Estado cumplidor (5) y en un Estado incumplidor (1, 2, 4 y 7), así como el resto de las preferencias (las poco probables), se pueden visualizar en Tabla 1 .

13. Se considera que la razón por la que el ciudadano preferiría la menor acción gubernamental es que el Estado no habría cumplido con su labor de garantizar la seguridad pública, combinado esto con una igual o menor preferencia/intención del ciudadano de participar.

Estado No.76 
Tabla 1. Combinaciones de preferencias ciudadanas

\begin{tabular}{|c|c|c|c|c|c|}
\hline 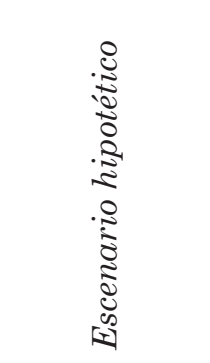 & 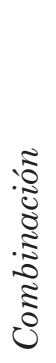 & 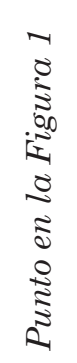 & 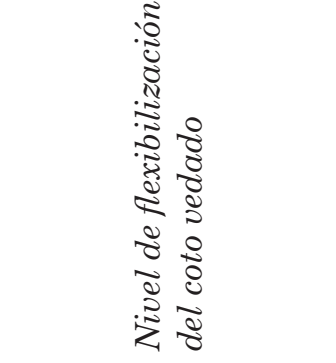 & 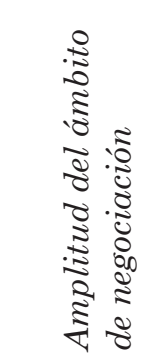 & 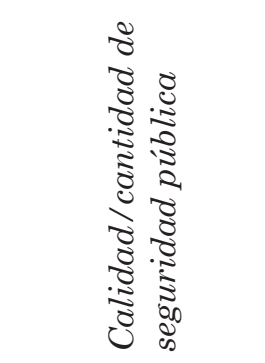 \\
\hline \multirow{4}{*}{$\begin{array}{l}\text { Estado } \\
\text { incumplidor }\end{array}$} & 1 & $\mathrm{a}$ & Flexible/extendido & Amplio & Perfecta/total \\
\hline & 2 & $\mathrm{~b}$ & Inflexible/rígido & Igual & $\begin{array}{l}\text { Excelente/ } \\
\text { mucha }\end{array}$ \\
\hline & 4 & $\mathrm{c}$ & Flexible/extendido & Amplio & $\begin{array}{l}\text { Muy buena/ } \\
\text { bastante }\end{array}$ \\
\hline & 7 & d & Flexible/extendido & Amplio & $\begin{array}{l}\text { Sobresaliente/ } \\
\text { suficiente }\end{array}$ \\
\hline \multirow{5}{*}{$\begin{array}{l}\text { Estado } \\
\text { cumplidor } \\
\text { Ambos* }\end{array}$} & 5 & e & Inflexible/rígido & Igual & Buena/regular \\
\hline & 3 & $\mathrm{n} / \mathrm{a}$ & Flexible/restringido & Reducido & Defectuosa/poca \\
\hline & 6 & $\mathrm{f}$ & Flexible/restringido & Reducido & $\begin{array}{l}\text { Mala/ } \\
\text { insuficiente }\end{array}$ \\
\hline & 8 & $\mathrm{n} / \mathrm{a}$ & Flexible/restringido & Reducido & $\begin{array}{l}\text { Pésima/muy } \\
\text { poca }\end{array}$ \\
\hline & 9 & $\mathrm{n} / \mathrm{a}$ & Flexible/restringido & Reducido & Nefasta/nada \\
\hline
\end{tabular}

* Produce combinaciones de preferencias poco probables.

Fuente: elaboración propia.

Como se puede observar, los niveles óptimos de calidad/ cantidad de seguridad pública corresponden a los niveles de flexibilización extendidos del coto vedado y a lo sumo a los rígidos, y también a niveles amplios o iguales del ámbito de negociación, ya que el ciudadano razonable (en un escenario ya sea de Estado incumplidor o cumplidor) prefiere involucrarse en las actividades tendientes a lograr la seguridad pública. Esos dos grupos forman dos curvas de indiferencia dentro de las cuales el ciudadano es impasible en cuanto a su preferencia en un determinado ambiente de seguridad pública, lo que puede visualizarse en la Figura 1. 
Figura 1. Curvas de indiferencia entre la acción gubernamental y la participación ciudadana

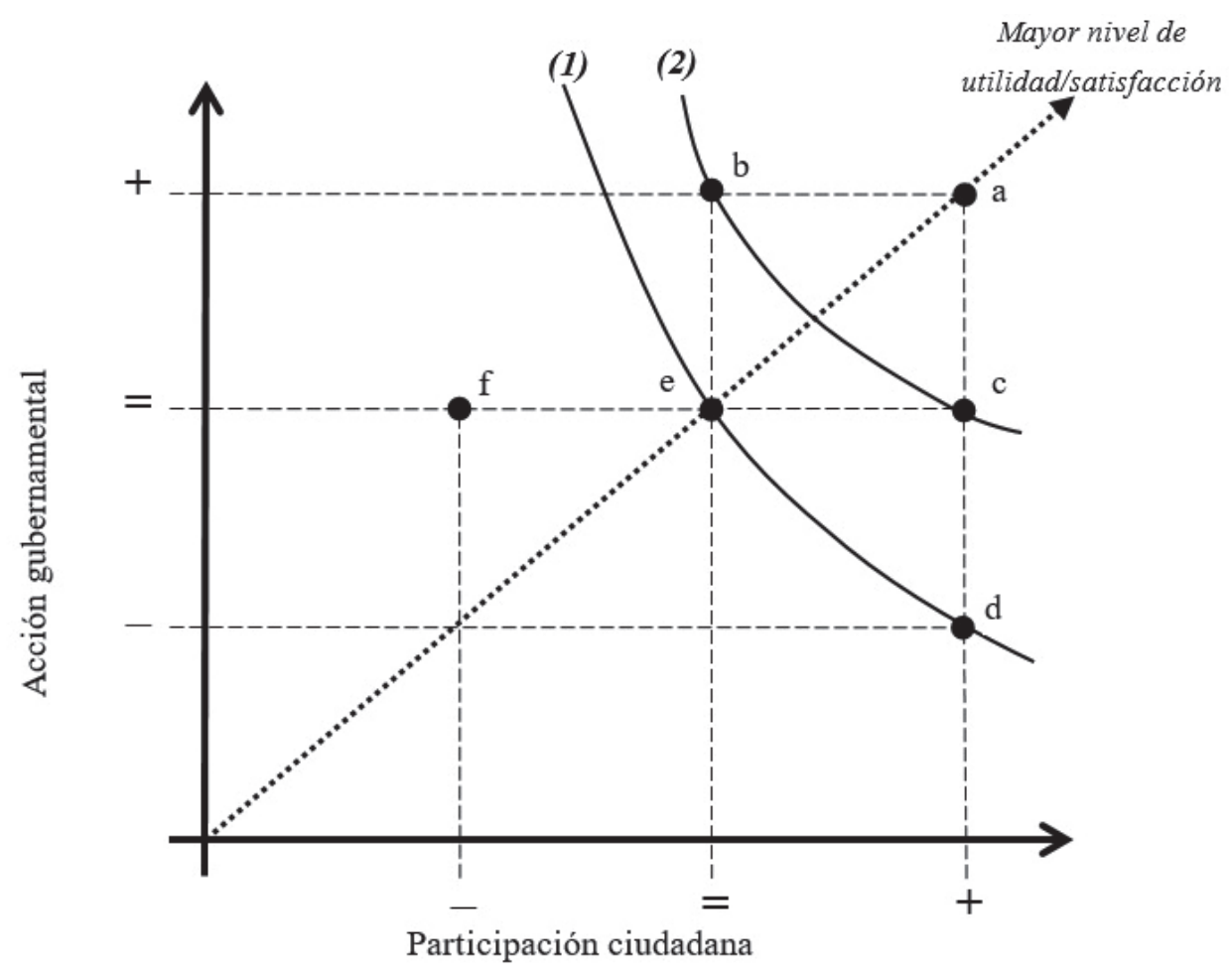

Fuente: elaboración propia.

En efecto, en esos grupos de combinaciones de preferencias del ciudadano razonable, la tensión entre la acción gubernamental (traducida como una intervención de los órganos e instituciones estatales) y la participación ciudadana (que resulta de su involucramiento en cuanto a la seguridad pública) trae como resultado la curva de indiferencia (1) formada por las preferencias de los puntos e y d, en la que ambos instrumentos (acción gubernamental y participación ciudadana) son idénticamente satisfactorios para la sociedad en función de la calidad y cantidad del bien que le proporcionan (seguridad pública) y dicha satisfacción está caracterizada por la utilidad que representan las variables involucradas en dicha curva. En cuanto alguna de las dos opciones aumenta, la otra disminuye, y las opciones que 
son indiferentes para el ciudadano se encuentran en la misma curva, tanto que si se desplaza a lo largo de una de las curvas entonces estaría dispuesto a aceptar una mayor acción gubernamental a cambio de una menor participación ciudadana, y si se desplaza en el otro sentido entonces estaría dispuesto a aceptar menor acción gubernamental por una mayor participación ciudadana, pero cualquier punto a lo largo de estas dos curvas le proporciona el mismo grado de satisfacción.

En caso de que el ciudadano tenga la opción de aumentar la acción gubernamental sin sacrificar su participación, entonces estará pasando a la curva de indiferencia (2) formada por las preferencias de los puntos b y c, con posibles graduaciones más precisas entre una y otra curva con las que se tendrían otras muchas curvas de indiferencia que no pueden incidir unas con otras en virtud de que cada una de ellas otorgaría el mismo nivel de utilidad.

$\mathrm{Al}$ ciudadano le conviene estar en cualquiera de los puntos de la curva de indiferencia, pero también le conviene estar en la curva más alta posible (en este caso, la (2)), ya que es mayor el nivel de satisfacción en ella, de tal manera que, desplazándose más hacia la posición a, podrá encontrar una mayor utilidad/satisfacción tendiente a la seguridad pública perfecta/total, pero también podría encontrarse a su vez en otra curva de indiferencia. Finalmente, el punto f es un ejemplo de una combinación de preferencias ciudadanas a la que no es indiferente el ciudadano, ya que no hay similitud en el grado de satisfacción reportado.

\section{Conclusión}

El objetivo principal del presente artículo fue analizar la relación existente entre seguridad pública, participación ciudadana y acción gubernamental en un ambiente de inseguridad pública en México. Se encontró que la acción 
gubernamental y la participación ciudadana son dos instrumentos cuyo objetivo es lograr el bien social denominado seguridad pública, sin embargo, el Gobierno mexicano no ha cumplido totalmente con el objetivo estatal de proporcionar esa seguridad a su población, lo que ha traído como consecuencia que la ciudadanía haya intervenido de diversas maneras en el fenómeno para solucionarlo, y esto demuestra que en un escenario de Estado incumplidor la preferencia es la participación ciudadana, esto con el objetivo de obtener ese bien social.

Derivado de ello, en México ha habido una tensión entre la acción gubernamental y la participación ciudadana emanada del grado de su intervención y participación en la solución del fenómeno, lo que evidencia una relación de interdependencia que va en función de dos escenarios: uno en el que el Estado no cumple con ese objetivo y otro en el que sí lo cumple. Por ello, en México es plausible que el ciudadano razonable prefiera que el coto vedado se flexibilice o, cuando mucho, se mantenga rígido, con la consecuente ampliación del ámbito de negociación o, al menos, su mantenimiento en iguales cantidades.

Finalmente, en ambos escenarios estatales la relación entre los dos instrumentos produce dos grupos de preferencias que forman dos curvas de indiferencia para el ciudadano razonable, ya que esos dos instrumentos son idénticamente satisfactorios para la sociedad en función de la calidad y cantidad de la seguridad pública que le proporcionan, y esa satisfacción está cimentada en la utilidad que representan las variables involucradas en dicha curva.

Bachrach, P.,y Botwinick,A. (1994).Análisis y reconstrucción Bibliografía teórica de la teoría participativa. En I. Pichardo Pagaza (Comp.), Política social y participación ciudadana (pp. I 1 I142). México: Mac. 
Bibliografía

Beliz, G. (20I2). Gobernar la seguridad ciudadana en América Latina y el Caribe.Amenazas, desafios y nudos estratégicos de gestión.Washington: Banco Interamericano de Desarrollo, Sector de Instituciones para el Desarrollo.

Carrillo, L. (20I0). El concepto kantiano de ciudadanía. Estudios de filosofia, (42), I03-12I. Recuperado de https:// aprendeenlinea.udea.edu.co/revistas/index.php/estudios_de_filosofia/article/view/I I 595

Centro de Opinión Pública, e Instituto para la Seguridad y la Democracia (2017). Ser policía en México: ¿qué rol asume la sociedad? Reporte ejecutivo de resultados. Recuperado de http://insyde.org.mx/wp-content/uploads/ 16-02-20 I 7-Presentación-estudio-Policias-completa-CopUVM-Insyde.pdf

Convención Americana sobre Derechos Humanos (22 de noviembre de 1969). Conferencia Especializada Interamericana sobre Derechos Humanos (B-32). Recuperado de http://www.oas.org/dil/esp/tratados_B-32_Convencion_Americana_sobre_Derechos_Humanos.htm

Diario Oficial de la Federación (26 de marzo de 2019). Decreto por el que se declaran reformadas y derogadas diversas disposiciones de la Constitución Política de los Estados Unidos Mexicanos, en materia de la reforma política de la Ciudad de México. Recuperado de http://www. dof.gob.mx/nota_detalle.php?codigo $=5424043 \&$ fecha $=29 / 01 / 2016$

Dworkin, R. (2004). Democracia deliberativa y derechos humanos. Barcelona: Gedisa.

El Mundo Internacional (I8 de octubre de 2016). Amputan las manos a siete ladrones en México. Recuperado de https://www.elmundo.es/internacional/20 I6/ I0/ I8/5806 I I 52468aebe4 I 38b4644.html

Excélsior (0I de noviembre de 2016). Asesinados en La Marquesa asaltaban con frecuencia: transportistas.

\section{0}


Recuperado de http://www.excelsior.com.mx/comuniBibliografía $\mathrm{dad} / 2016 / \mathrm{I}$ I/0 I/I I 25583

Flores, E. (20I3). Autodefensas desplazan a la autoridad y asumen seguridad en Apaxtla, Guerrero. Proceso. Recuperado de http://www.proceso.com.mx/359206/ autodefensas-asumen-seguridad-en-apaxtla-guerrero

García Ramírez, S. (2002). En torno a la seguridad pública. Desarrollo penal y evolución del delito. En P.J.Peñaloza, y M.A. Garza Salinas (Coords.), Los desafios de la seguridad pública en México (Pp. 8I-98). México: Universidad Iberoamericana, UNAM, PGR.

Garzón, E. (2003).Algunas consideraciones sobre la posibilidad de asegurar la vigencia del "coto vedado" a nivel internacional. Derechos y libertades. Revista del Instituto de Derechos Humanos Bartolomé de las Casas, 8(I2), 57-70. Recuperado de https://e-archivo.uc3m.es/handle/I0016/37/3\#preview

Garzón, E. (2005). El consenso democrático: fundamento y límites del papel de las minorías. Isonomía. Revista de teoría y filosofia del derecho, (I2), 6-34. Recuperado de http:// www.cervantesvirtual.com/nd/ark:/5985 I/bmcks 725

Garzón, E. (20I I). Las muletas morales del ciudadano en la democracia. Revista del Instituto de la Judicatura Federal, (32), 69-86. Recuperado de https://www.ijf.cjf.gob.mx/ Sitio20 I 6/include/sections/revista/32/RIJF\%20No\%20 32\%20INTERIORES-ERNESTO\%20GARZON.pdf

González Uribe, H. (1995). Teoría política. México: Porrúa.

Guerrero, E. (2016). La inseguridad 20I3-20I5. Nexos. Recuperado de http://www.nexos.com.mx/?p=27269

Hobbes, T. (1984). Leviatán o la materia, forma y poder de una república eclesiástica y civil. Madrid: Sarpe.

Instituto Federal Electoral (2013). Estudio censal de la participación ciudadana en las elecciones federales de 20I 2. Recuperado de http://www.ine.mx/docs/IFE-v2/DECEYEC/ 

ridad pública y justicia. Recuperado de http://www.beta. inegi.org.mx/temas/incidencia/

Instituto Nacional Electoral (20I5). Informe país sobre la calidad de la ciudadanía en México. Recuperado de http:// www.ine.mx/archivos2/s/DECEYEC/EducacionCivica/ Resumen_Ejecutivo_23nov.pdf

Martínez Elorriaga, E. (19 de febrero de 2014). En Michoacán la inseguridad se redujo 70\%: Osorio Chong. La Jornada. Recuperado de http://www.jornada.unam. $\mathrm{mx} / 20$ I4/02/I9/politica/0 I $\mathrm{n}$ I pol

Mujica, P. (2008). El concepto de participación ciudadana y su aplicación en las políticas públicas. En P.Valenzuela, y C. Delpiano (Eds.), Manual de participación ciudadana (pp. I I-48). Santiago de Chile: Corporación Participa.

Rawls, J. (1993). Political liberalism. Nueva York: Columbia University Press.

Real Academia Española (2017a). Seguridad. Diccionario de la Real Academia Española. Recuperado de https://dle. rae.es/?id=XTrlaQd

Real Academia Española (2017b). Público. Diccionario de la Real Academia Española. Recuperado de https://dle.rae. es/?id=UYbbTs8

Real Academia Española (20I8). Bien. Diccionario de la Real Academia Española. Recuperado de https://dle.rae. es/?id=5TkGdE0

Santiago, C. (2003). La constitución de la democracia deliberativa. Barcelona: Gedisa.

Univisión (29 de diciembre de 2013).Autodefensas en México se extienden: controlan 28 municipios de Michoacán. Recuperado de http://www.univision.com/noticias/ noticias-de-mexico/autodefensas-en-mexico-se-extienden-controlan-28-municipios-de-michoacan 